\title{
FUNCTIONS OF LEAST GRADIENT AND 1-HARMONIC FUNCTIONS
}

\author{
JOSÉ M. MAZÓN, JULIO D. ROSSI, AND SERGIO SEGURA DE LEÓN
}

\begin{abstract}
In this paper we find the Euler-Lagrange equation corresponding to functions of least gradient. It turns out that this equation can be identified with the 1-Laplacian. Moreover, given a Lipschitz domain, $\Omega$, we prove that there exists a function of least gradient in $\Omega$ that extends every datum belonging to $L^{1}(\partial \Omega)$. We show, as well, the non uniqueness of solutions in the case of discontinuous boundary values.
\end{abstract}

\section{INTRODUCTION}

Let $\Omega$ be an open bounded subset of $\mathbb{R}^{N}$ with Lipschitz boundary and a function $h: \partial \Omega \rightarrow \mathbb{R}$. The least gradient problem with a boundary datum given by $h$ is the problem of minimizing

$$
\min \left\{\int_{\Omega}|D u|: u \in B V(\Omega), u=h \text { on } \partial \Omega\right\} .
$$

Here $B V(\Omega)$ is the space of bounded variation functions, that is, the space of functions $u \in L^{1}(\Omega)$ such that the distributional gradient $D u$ is a bounded Radon measure with finite total variation $|D u|$. The minimizers of problem (1.1) are called functions of least gradient. Among the first works that study this issue we highlight [10] and [5].

The coarea formula (see [1])

$$
\int_{\Omega}|D u|=\int_{-\infty}^{+\infty} \operatorname{Per}(\{u \geq t\}, \Omega) d t
$$

where $\operatorname{Per}(\{u \geq t\}, \Omega):=\left|D \chi_{\{u \geq t\}}\right|$ denotes the perimeter of the superlevel set $\{x \in \Omega: u(x) \geq t\}$, connects the functions of least gradient with the theory of parametric minimal surfaces. Indeed, in [5] Bombieri, De Giorgi and Giusti showed that the superlevel sets of a continuous function of least gradient are area-minimizing, that is, the characteristic functions of those sets are functions of least gradient. Conversely, Sternberg, Williams and Ziemmer in [11] (see also [12]) proved the existence of a function of least gradient by explicitly constructing each of its superlevel sets in such a way that they are area-minimizing and reflect the boundary condition. More precisely, they proved that for $\Omega \subset \mathbb{R}^{N}$ a bounded Lipschitz domain such that $\partial \Omega$ has non-negative mean curvature (in a weak sense) and is not

Key words and phrases. Functions of Least Gradient, 1-Laplacian.

Mathematics Subject Classification 2010 : 35J75, 35J20, 35J92, 35J25. 
locally are-minimizing and $h \in C(\partial \Omega)$, there exists a unique function of least gradient $u \in B V(\Omega) \cap C(\bar{\Omega})$ such that $u=h$ on $\partial \Omega$.

Juutinen in [8] proved that the unique function of least gradient $u$ obtained in the previously mentioned result is the uniform limit as $p \searrow 1$ of the unique $p$-harmonic function in $\Omega$ with Dirichlet datum $h$, that is, a solution to $\operatorname{div}\left(\left|\nabla u_{p}\right|^{p-2} \nabla u_{p}\right)=0$, satisfying $u_{p}=h$ on $\partial \Omega$. As a consequence he obtains that functions of least gradient satisfy

$$
-|\nabla u|^{2} \Delta u+D^{2} u \nabla u \cdot \nabla u=0
$$

in the viscosity sense. Hence, functions of least gradient are viscosity solutions to (1.2). However, as Juutinen pointed out, the converse is not true, viscosity solutions to (1.2) are not necessarily least gradient functions, as shown in [13, Example 3.6]. Therefore, equation (1.2) is not the EulerLagrange equation of the minimization problem (1.1).

Our main aim here is to identify the Euler-Lagrange equation for (1.1). More precisely, the purpose of this paper is to establish the equivalence between functions of least gradient and solutions to the Dirichlet problem for the 1-Laplacian, $-\operatorname{div}\left(\frac{D u}{|D u|}\right)=0$.

Our main result is the following:

\section{Theorem 1.1.}

1) Let $h \in L^{1}(\partial \Omega)$. For each $v \in B V(\Omega)$ satisfying $\left.v\right|_{\partial \Omega}=h$ the following conditions are equivalent:

(i) $v$ is a solution to

$$
\begin{cases}-\operatorname{div}\left(\frac{D u}{|D u|}\right)=0, & \text { in } \Omega ; \\ u=h, & \text { on } \partial \Omega ;\end{cases}
$$

in the sense of Definition 2.3 below.

(ii) $v$ is a function of least gradient, that is, a minimizer of (1.1).

2) For every $h \in L^{1}(\partial \Omega)$ there exists a solution to (1.3).

In addition, we show that functions of least gradient may not be unique if the boundary datum $h$ is in $L^{1}(\partial \Omega) \backslash C(\partial \Omega)$.

We present a proof of this result in the next section. Now, we make some remarks on our results. In particular, we describe briefly how our results are related to previous works.

The approach used in [11] and [12] is applicable whenever the solution is uniquely determined by its level sets. This procedure provides a unique solution for continuous data. From our results we conclude that this procedure is no longer applicable if the boundary datum is not continuous (since we may have nonuniqueness of solutions).

It was assumed in [11] and [12] that $\partial \Omega$ has nonnegative mean curvature and that it is locally area-minimizing. Furthermore, if either condition fails, the authors of these references show that there exists a boundary datum for 
which the corresponding least gradient extension problem has no continuous solution. It is worth to remark that the only assumption we make on the boundary of the domain is to be Lipschitz-continuous.

We also remark that we deal with functions of least gradient that are not necessarily continuous, this allows us to consider more general boundary data $h \in L^{1}(\partial \Omega)$.

Our approach to show existence, as in [8], is also based in taking the limit as $p \searrow 1$ of $p$-harmonic functions. However, our ideas and methods are very different. We are based on fine properties of bounded variation functions. Our ideas allow us to find that the equation that characterizes functions of least gradient is the 1-Laplacian. Note that this characterization also holds for continuous data. We have that a function, whose trace is the datum $h$, is a continuous least gradient function if and only if it is a continuous solution to the 1-Laplacian.

Note that one of the major difficulties to define a solution to the 1Laplacian is to give a sense to $\frac{D u}{|D u|}$, especially where $D u$ vanishes. Another difficulty is to make sense of the boundary condition for general $h \in L^{1}(\partial \Omega)$, since it does not necessarily hold in the sense of traces. Following [2], we overcome these difficulties by means of a bounded vector field $\mathbf{z}$ which plays the role of $\frac{D u}{|D u|}$ and by considering a weak sense for the boundary condition (see (2.13) below). Therefore, we will deal in this paper with weak solutions to problem (1.3) in the sense of Definition 2.3.

\section{Proof of Theorem 1.1.}

2.1. Preliminaries. For our purposes we need to recall the Green formula obtained by Anzellotti in [4]. Consider

$$
X_{N}(\Omega)=\left\{\mathbf{z} \in L^{\infty}\left(\Omega, \mathbb{R}^{N}\right): \operatorname{div}(\mathbf{z}) \in L^{N}(\Omega)\right\} .
$$

If $\mathbf{z} \in X_{N}(\Omega)$ and $w \in B V(\Omega)$, we define $(\mathbf{z}, D w): C_{0}^{\infty}(\Omega) \rightarrow \mathbb{R}$ by the formula

$$
\langle(\mathbf{z}, D w), \varphi\rangle:=-\int_{\Omega} w \varphi \operatorname{div}(\mathbf{z}) d x-\int_{\Omega} w \mathbf{z} \cdot \nabla \varphi d x .
$$

In [4] (see also [3, Corollary C.7, C.16]) it is proved the following result.

Proposition The distribution $(\mathbf{z}, D w)$ is actually a Radon measure with finite total variation.

Moreover, the measures $(\mathbf{z}, D w),|(\mathbf{z}, D w)|$ are absolutely continuous with respect to the measure $|D w|$ and

$$
\left|\int_{B}(\mathbf{z}, D w)\right| \leq \int_{B}|(\mathbf{z}, D w)| \leq\|\mathbf{z}\|_{L^{\infty}(U)} \int_{B}|D w|
$$

for all Borel sets $B$ and for all open sets $U$ such that $B \subset U \subset \Omega$.

In [4], a weak trace on $\partial \Omega$ of the normal component of $\mathbf{z} \in X_{N}(\Omega)$ is defined. More precisely, it is proved that there exists a linear operator 
$\gamma: \mathbf{z} \in X_{N}(\Omega) \rightarrow L^{\infty}(\partial \Omega)$ such that

$$
\|\gamma(\mathbf{z})\|_{\infty} \leq\|\mathbf{z}\|_{\infty}
$$

and

$$
\gamma(\mathbf{z})(x)=\mathbf{z}(x) \cdot \nu(x) \quad \text { for all } x \in \partial \Omega \text { if } \mathbf{z} \in C^{1}\left(\bar{\Omega}, \mathbb{R}^{N}\right) .
$$

We will denote $\gamma(\mathbf{z})(x)$ by $[\mathbf{z}, \nu](x)$. Moreover, the following Green's formula, that relates the function $[\mathbf{z}, \nu]$ and the measure $(\mathbf{z}, D w)$, for $\mathbf{z} \in X_{N}(\Omega)$ and $w \in B V(\Omega)$, is established

$$
\int_{\Omega} w \operatorname{div}(\mathbf{z}) d x+\int_{\Omega}(\mathbf{z}, D w)=\int_{\partial \Omega}[\mathbf{z}, \nu] w d \mathcal{H}^{N-1} .
$$

Here and in what follows, $\mathcal{H}^{N-1}$ will denote the $N$-1-dimensional Hausdorff measure.

\subsection{Functions of Least Gradient and the 1-Laplacian.}

Definition 2.1. We say that $u \in B V(\Omega)$ is a function of least gradient if

$$
\int_{\Omega}|D u| \leq \int_{\Omega}|D(u+v)|
$$

for all $v \in B V(\Omega)$ such that $\operatorname{spt} v \subset \Omega$.

REMARK 2.2. Definition 2.1 is frequently used in the literature (see, for instance, [5]). Nevertheless, it was proved in [12, Theorem 2.2] that this definition is equivalent to require that

$$
\int_{\Omega}|D u| \leq \int_{\Omega}|D v|
$$

for all $v \in B V(\Omega)$ such that $\left.v\right|_{\partial \Omega}=\left.u\right|_{\partial \Omega}$.

It is well known (see [6] or [2]) that for $h \in L^{1}(\partial \Omega)$ the relaxed energy functional associated to problem (1.1) is the functional

$$
\Phi_{h}: L^{\frac{N}{N-1}}(\Omega) \rightarrow(-\infty,+\infty]
$$

defined by

$$
\Phi_{h}(u)= \begin{cases}\int_{\Omega}|D u|+\int_{\partial \Omega}|u-h| d \mathcal{H}^{N-1} & \text { if } u \in B V(\Omega), \\ +\infty & \text { if } u \in L^{\frac{N}{N-1}}(\Omega) \backslash B V(\Omega) .\end{cases}
$$

The functional $\Phi_{h}$ is convex and lower semicontinuous in $L^{\frac{N}{N-1}}(\Omega)$. In order to deal with the variational problem

$$
\min _{w \in B V(\Omega)} \Phi_{h}(w)
$$


we will analyze the subdifferential of $\Phi_{h}$. For any $u \in L^{\frac{N}{N-1}}(\Omega)$ we can define the subgradient of $u$ in the duality $\left(L^{\frac{N}{N-1}}(\Omega), L^{N}(\Omega)\right)$ as $\partial \Phi_{h}(u):=\left\{\begin{array}{c}f \in L^{N}(\Omega): \Phi_{h}(w)-\Phi_{h}(u) \geq \int_{\Omega} f(x)(w(x)-u(x)) d x \\ \forall w \in L^{\frac{N}{N-1}}(\Omega)\end{array}\right\}$.

We have

$$
\Phi_{h}(u)=\min _{w \in B V(\Omega)} \Phi_{h}(w) \Longleftrightarrow 0 \in \partial \Phi_{h}(u) .
$$

Using some of the ideas introduced in [2] to study the Dirichlet problem for the Total Variation Flow, we are going to find a characterization of the minimizer $u$ of problem (2.7).

We begin by recalling the continuous embedding

$$
B V\left(\mathbb{R}^{N}\right) \hookrightarrow L^{\frac{N}{N-1}}\left(\mathbb{R}^{N}\right) .
$$

On the other hand, since $\Omega$ has Lipschitz boundary, if $u$ belongs to $B V(\Omega)$, then the function

$$
u_{0}= \begin{cases}u, & \text { in } \Omega \\ 0, & \text { in } \mathbb{R}^{N} \backslash \Omega\end{cases}
$$

belongs to $B V\left(\mathbb{R}^{N}\right)$ and

$$
\left|D u_{0}\right|\left(\mathbb{R}^{N}\right)=\int_{\partial \Omega}|u| d \mathcal{H}^{N-1}+|D u|(\Omega) .
$$

Combining these two facts, it follows that the usual norm in $B V(\Omega)$ is equivalent to the one given by

$$
\|u\|=\int_{\Omega}|D u|+\int_{\partial \Omega}|u| d \mathcal{H}^{N-1} .
$$

Next, we will consider de Dirichlet problem for the 1-Laplacian:

$$
\begin{cases}-\operatorname{div}\left(\frac{D u}{|D u|}\right)=0, & \text { in } \Omega \\ u=h, & \text { on } \partial \Omega .\end{cases}
$$

Functions satisfying the PDE in (2.10) are called 1-harmonic functions. Note that in regions where $u$ is smooth and $D u$ does not vanish, $\operatorname{div}\left(\frac{D u}{|D u|}\right)$ is the scalar mean curvature of the level sets of $u$. So this PDE asserts that each level surface of $u$ has mean curvature zero. Following the characterization of the subdifferential of $\Phi_{h}$ given in [2], we give the following definition. 
Definition 2.3. We will say that $u \in B V(\Omega)$ is a solution to problem (2.10) if there exists a vector field $\mathbf{z} \in L^{\infty}\left(\Omega ; \mathbb{R}^{N}\right)$, with $\|\mathbf{z}\|_{\infty} \leq 1$, satisfying

$$
\begin{aligned}
& -\operatorname{div}(\mathbf{z})=0, \quad \text { in } \mathcal{D}^{\prime}(\Omega), \\
& \quad(\mathbf{z}, D u)=|D u|, \\
& {[\mathbf{z}, \nu] \in \operatorname{sign}(h-u), \quad \mathcal{H}^{N-1} \text {-a.e. on } \partial \Omega .}
\end{aligned}
$$

With this definition in mind we can state the following result.

Theorem 2.4. For each $h \in L^{1}(\partial \Omega)$ there exists a solution to problem (2.10).

Proof. Suppose first that $h \in W^{1 / 2,2}(\partial \Omega)$, so that there exists $v \in W^{1,2}(\Omega)$ such that $\left.v\right|_{\partial \Omega}=h$. Then $h \in W^{1-1 / p, p}(\partial \Omega)$ for all $1<p \leq 2$. We begin by denoting

$$
W_{h}^{1, p}(\Omega):=\left\{u \in W^{1, p}(\Omega):\left.u\right|_{\partial \Omega}=h \quad \mathcal{H}^{N-1} \text { - a.e. on } \partial \Omega\right\},
$$

so that $v \in W_{h}^{1, p}(\Omega)$ for all $1<p \leq 2$.

For each $1<p \leq 2$, consider the problem

$$
\begin{cases}-\operatorname{div}\left(\left|\nabla u_{p}\right|^{p-2} \nabla u_{p}\right)=0, & \text { in } \Omega ; \\ u_{p}=h, & \text { on } \partial \Omega ;\end{cases}
$$

It is well-known (see for instance [7]) that there exists $u_{p} \in W_{h}^{1, p}(\Omega)$ which is a solution to problem (2.14), that is, which satisfies

$$
\int_{\Omega}\left|\nabla u_{p}\right|^{p-2} \nabla u_{p} \cdot \nabla\left(w-u_{p}\right) d x=0,
$$

for every $w \in W_{h}^{1, p}(\Omega)$.

Fixed $w=v \in W_{h}^{1, p}(\Omega)$ in (2.15) and applying Hölder's inequality we obtain

$$
\begin{aligned}
\int_{\Omega}\left|\nabla u_{p}\right|^{p} d x=\int_{\Omega}\left|\nabla u_{p}\right|^{p-2} \nabla & u_{p} \cdot \nabla v d x \\
\leq & \left(\int_{\Omega}\left|\nabla u_{p}\right|^{p} d x\right)^{1 / p^{\prime}}\left(\int_{\Omega}|\nabla v|^{p} d x\right)^{1 / p} .
\end{aligned}
$$

Hence,

$$
\begin{aligned}
\left(\int_{\Omega}\left|\nabla u_{p}\right|^{p} d x\right)^{1 / p} & \leq\left(\int_{\Omega}|\nabla v|^{p} d x\right)^{1 / p} \\
& \leq\left(\int_{\Omega}|\nabla v|^{2} d x\right)^{1 / 2} \mathcal{L}^{N}(\Omega)^{(2-p) / 2} \\
& \leq\left(\int_{\Omega}|\nabla v|^{2} d x\right)^{1 / 2}\left(1+\mathcal{L}^{N}(\Omega)\right)^{1 / 2} .
\end{aligned}
$$


and so

$$
\left(\int_{\Omega}\left|\nabla u_{p}\right|^{p} d x\right)^{1 / p} \leq C,
$$

where $C$ is a constant that does not depend on $p$.

Thus, on the one hand,

$$
\int_{\Omega}\left|\nabla u_{p}\right|^{p} d x \leq C^{p} \leq(1+C)^{2}=M_{1} \quad \forall 1<p \leq 2,
$$

where $M_{1}$ does not depend on $p$. On the other hand, applying Hölder's inequality, we also have that

$$
\int_{\Omega}\left|\nabla u_{p}\right| d x \leq C \mathcal{L}^{N}(\Omega)^{1-\frac{1}{p}} \leq C\left(1+\mathcal{L}^{N}(\Omega)\right)^{1 / 2}=M_{2} \quad \forall 1<p \leq 2,
$$

where $M_{2}$ does not depend on $p$. Moreover, since $\left.u_{p}\right|_{\partial \Omega}=h$, it follows that

$$
\left\|u_{p}\right\|=\int_{\Omega}\left|\nabla u_{p}\right|+\int_{\partial \Omega}\left|u_{p}\right| d \mathcal{H}^{N-1} \leq M_{2}+\int_{\partial \Omega}|h| \mathcal{H}^{N-1} .
$$

Therefore, $\left\{u_{p}\right\}_{p>1}$ is bounded in $B V(\Omega)$ and by the compact embedding, we may extract a subsequence (not relabeled) and find $u \in B V(\Omega)$ such that

$$
\lim _{p \rightarrow 1+} u_{p}=u \text { strongly in } L^{r}(\Omega), \forall 1 \leq r<\frac{N}{N-1}, \text { and a.e. }
$$

From (2.17), working as in the proof of $[2$, Proposition 3] (see also [9, Proposition 4.1]), we can prove that there exists $\mathbf{z} \in L^{\infty}\left(\Omega ; \mathbb{R}^{N}\right)$ such that $\|\mathbf{z}\|_{\infty} \leq 1$ and

$$
\left|\nabla u_{p}\right|^{p-2} \nabla u_{p} \rightarrow \mathbf{z}, \quad \text { weakly in } L^{q}(\Omega) \quad \forall q<\infty .
$$

Given $\varphi \in C_{0}^{\infty}(\Omega)$, taking $w=u_{p}-\varphi$ as test function in (2.15), we have

$$
\int_{\Omega}\left|\nabla u_{p}\right|^{p-2} \nabla u_{p} \cdot \nabla \varphi d x=0 .
$$

Then, taking limits as $p \rightarrow 1^{+}$, by $(2.21)$, we get

$$
\int_{\Omega} \mathbf{z} \cdot \nabla \varphi=0
$$

and consequently,

$$
\operatorname{div}(\mathbf{z})=0, \quad \text { in } \mathcal{D}^{\prime}(\Omega) .
$$

On the other hand, given $0 \leq \varphi \in C_{0}^{\infty}(\Omega)$, taking $w=u_{p}-u_{p} \varphi$ as test function in (2.15), we have

$$
\int_{\Omega} u_{p}\left|\nabla u_{p}\right|^{p-2} \nabla u_{p} \cdot \nabla \varphi d x+\int_{\Omega} \varphi\left|\nabla u_{p}\right|^{p-2} \nabla u_{p} \cdot \nabla u_{p} d x=0 .
$$

Having in mind $(2.20),(2.21)$ and the lower semi-continuity of the total variation, we can past to the limit as $p \rightarrow 1^{+}$in the last equality to get

$$
\int_{\Omega} u \mathbf{z} \cdot \varphi+\int_{\Omega} \varphi|D u| \leq 0,
$$


from where it follows

$$
\int_{\Omega} \varphi|D u| \leq-\int_{\Omega} u \mathbf{z} \cdot \varphi=\langle(\mathbf{z}, D u), \varphi
$$

and consequently

$$
|D u| \leq(\mathbf{z}, D u) \quad \text { as measures. }
$$

Therefore, it follows from $\|\mathbf{z}\|_{\infty} \leq 1$ that

$$
|D u|=(\mathbf{z}, D u) \quad \text { as measures. }
$$

Taking again $w=v$ in (2.15) and applying Young's inequality, we obtain

$$
\begin{aligned}
\int_{\Omega} \mid & \nabla u_{p}\left|d x+\int_{\partial \Omega}\right| u_{p}-h \mid d \mathcal{H}^{N-1} \\
& =\int_{\Omega}\left|\nabla u_{p}\right| d x \leq \frac{1}{p} \int_{\Omega}\left|\nabla u_{p}\right|^{p} d x+\frac{p-1}{p} \mathcal{L}^{N}(\Omega) \\
& =\frac{1}{p} \int_{\Omega}\left|\nabla u_{p}\right|^{p-2} \nabla u_{p} \cdot \nabla v d x+\frac{p-1}{p} \mathcal{L}^{N}(\Omega) .
\end{aligned}
$$

Then, by the lower semi-continuity of $\Phi_{h}$, and applying Green's formula, we get

$$
\int_{\Omega}|D u|+\int_{\partial \Omega}|u-h| d \mathcal{H}^{N-1} \leq \int_{\Omega} \mathbf{z} \cdot \nabla v d x=\int_{\partial \Omega} h[\mathbf{z}, \nu] d \mathcal{H}^{N-1} .
$$

Now, applying again Green's formula and (2.22), we have

$$
\begin{aligned}
\int_{\Omega}|D u|+\int_{\partial \Omega}|u-h| d \mathcal{H}^{N-1} \\
\leq \int_{\partial \Omega}(h-u)[\mathbf{z}, \nu] d \mathcal{H}^{N-1}+\int_{\partial \Omega} u[\mathbf{z}, \nu] d \mathcal{H}^{N-1} \\
=\int_{\partial \Omega}(h-u)[\mathbf{z}, \nu] d \mathcal{H}^{N-1}+\int_{\Omega}(\mathbf{z}, D u) \\
=\int_{\partial \Omega}(h-u)[\mathbf{z}, \nu] d \mathcal{H}^{N-1}+\int_{\Omega}|D u| .
\end{aligned}
$$

Thus,

from where it follows that

$$
\int_{\partial \Omega}|u-h| d \mathcal{H}^{N-1} \leq \int_{\partial \Omega}(h-u)[\mathbf{z}, \nu] d \mathcal{H}^{N-1}
$$

$$
[\mathbf{z}, \nu] \in \operatorname{sign}(h-u), \quad \mathcal{H}^{N-1} \text {-a.e. on } \partial \Omega,
$$

and we concludes the proof in this case.

Assume now the general case, that is, $h \in L^{1}(\partial \Omega)$. Take $v_{n} \in W^{1,2}(\Omega)$, such that $h_{n}:=\left.v_{n}\right|_{\partial \Omega} \rightarrow h$ in $L^{1}(\partial \Omega)$. From the previous arguments, there exists $u_{n} \in B V(\Omega)$ and $\mathbf{z}_{n} \in L^{\infty}\left(\Omega ; \mathbb{R}^{N}\right)$ with $\left\|\mathbf{z}_{n}\right\|_{\infty} \leq 1$ such that

$$
\begin{gathered}
\operatorname{div}\left(\mathbf{z}_{n}\right)=0 \quad \text { in } \mathcal{D}^{\prime}(\Omega), \\
\left(\mathbf{z}_{n}, D u_{n}\right)=\left|D u_{n}\right|
\end{gathered}
$$


and

$$
\left[\mathbf{z}_{n}, \nu\right] \in \operatorname{sign}\left(h_{n}-u_{n}\right), \quad \mathcal{H}^{N-1} \text {-a.e. on } \partial \Omega .
$$

We can assume, without loss of generality, that

$$
\mathbf{z}_{n} \rightarrow \mathbf{z} \quad \text { weakly* in } L^{\infty}\left(\Omega, \mathbb{R}^{N}\right),
$$

which implies $\operatorname{div}(\mathbf{z})=0$.

Given $v \in W^{1,1}(\Omega)$, by (2.23), using Green's formula we have

$$
\int_{\Omega}\left(\mathbf{z}_{n}, D\left(u_{n}-v\right)\right)=\int_{\partial \Omega}\left(u_{n}-v\right)\left[\mathbf{z}_{n}, \nu\right] d \mathcal{H}^{N-1},
$$

from where it follows, having in mind (2.24) and (2.25), that

$$
\begin{aligned}
\int_{\Omega}\left|D u_{n}\right|+\int_{\partial \Omega}\left|u_{n}-h_{n}\right| d \mathcal{H}^{N-1} \\
\quad=\int_{\Omega} \mathbf{z}_{n} \cdot \nabla v d x+\int_{\partial \Omega}\left(h_{n}-v\right)\left[\mathbf{z}_{n}, \nu\right] d \mathcal{H}^{N-1} .
\end{aligned}
$$

Then, applying Green's formula we get

$$
\begin{aligned}
\int_{\Omega}\left|D u_{n}\right|+ & \int_{\partial \Omega}\left|u_{n}-h_{n}\right| d \mathcal{H}^{N-1} \\
& =\int_{\partial \Omega} v\left[\mathbf{z}_{n}, \nu\right] d \mathcal{H}^{N-1}+\int_{\partial \Omega}\left(h_{n}-v\right)\left[\mathbf{z}_{n}, \nu\right] d \mathcal{H}^{N-1} \\
& \leq \int_{\partial \Omega}\left|h_{n}\right| d \mathcal{H}^{N-1} \leq C,
\end{aligned}
$$

and so

$$
\int_{\Omega}\left|D u_{n}\right|+\int_{\partial \Omega}\left|u_{n}\right| d \mathcal{H}^{N-1} \leq 2 \int_{\partial \Omega}\left|h_{n}\right| d \mathcal{H}^{N-1} \leq C .
$$

Therefore, by compact embedding there exists $u \in B V(\Omega)$ such that, taking a subsequence, if necessary,

$$
\lim _{n \rightarrow \infty} u_{n}=u \quad \text { strongly in } L^{1}(\Omega) \text { and a.e. }
$$

Now, from (2.27), we obtain that

$$
\begin{aligned}
\int_{\Omega}\left|D u_{n}\right|+ & \int_{\partial \Omega}\left|u_{n}-h\right| d \mathcal{H}^{N-1} \\
& \leq \int_{\Omega} \mathbf{z}_{n} \cdot \nabla v d x+\int_{\partial \Omega}\left|v-h_{n}\right| d \mathcal{H}^{N-1}+\int_{\partial \Omega}\left|h_{n}-h\right| d \mathcal{H}^{N-1} .
\end{aligned}
$$

Then, by the lower semi-continuity of $\Phi_{h}$ and (2.26), taking limits as $n \rightarrow \infty$, we deduce that

$$
\int_{\Omega}|D u|+\int_{\partial \Omega}|u-h| d \mathcal{H}^{N-1} \leq \int_{\Omega} \mathbf{z} \cdot \nabla v d x+\int_{\partial \Omega}|v-h| d \mathcal{H}^{N-1},
$$

holds for every $v \in W^{1,1}(\Omega)$. 
Using [3, Theorem B.3] and [3, Lemma C.8], we know that there exists a sequence $v_{n} \in W^{1,1}(\Omega)$ such that

$$
\begin{aligned}
& v_{n} \rightarrow u \quad \text { in } L^{1}(\Omega), \\
& \int_{\Omega}\left|\nabla v_{n}\right| d x \rightarrow \int_{\Omega}|D u|, \\
& \int_{\Omega} \mathbf{z} \cdot \nabla v_{n} d x=\int_{\Omega}\left(\mathbf{z}, D v_{n}\right) \rightarrow \int_{\Omega}(\mathbf{z}, D u) .
\end{aligned}
$$

and $\left.v_{n}\right|_{\partial \Omega}=\left.u\right|_{\partial \Omega}, \forall n \in \mathbb{N}$. Then, by (2.29), taking $v=v_{n}$ in (2.28) and taking limit as $n \rightarrow \infty$, we obtain that

$$
\int_{\Omega}|D u|+\int_{\partial \Omega}|u-h| d \mathcal{H}^{N-1} \leq \int_{\Omega}(\mathbf{z}, D u)+\int_{\partial \Omega}|u-h| d \mathcal{H}^{N-1} .
$$

Hence,

from where it follows that

$$
\int_{\Omega}|D u| \leq \int_{\Omega}(\mathbf{z}, D u) \leq \int_{\Omega}|D u|
$$

$$
|D u|=(\mathbf{z}, D u) \quad \text { as measures. }
$$

On the other hand, by [4, Lemma 5.5] (see also [3, Lemma C.1]), there exist $w_{n} \in W^{1,1}(\Omega)$ satisfying:

$$
\begin{gathered}
\left.w_{n}\right|_{\partial \Omega}=h \quad \forall n \in \mathbb{N}, \\
\int_{\Omega}\left|\nabla w_{n}\right| d x \leq \int_{\partial \Omega}|h| d \mathcal{H}^{N-1}+\frac{1}{n} \quad \forall n \in \mathbb{N}, \\
\left\|w_{n}\right\|_{1} \leq \frac{1}{n}, \quad \forall n \in \mathbb{N} .
\end{gathered}
$$

Taking $v=w_{n}$ in (2.28), we have

$$
\begin{gathered}
\int_{\Omega}(\mathbf{z}, D u)+\int_{\partial \Omega}|u-h| d \mathcal{H}^{N-1}=\int_{\Omega}|D u|+\int_{\partial \Omega}|u-h| d \mathcal{H}^{N-1} \\
\leq \int_{\Omega} \mathbf{z} \cdot \nabla w_{n} d x=\int_{\partial \Omega} h[\mathbf{z}, \nu] d \mathcal{H}^{N-1} .
\end{gathered}
$$

Then, applying Green's formula,

$$
\int_{\partial \Omega} u[\mathbf{z}, \nu] d \mathcal{H}^{N-1}+\int_{\partial \Omega}|u-h|[\mathbf{z}, \nu] d \mathcal{H}^{N-1} \leq \int_{\partial \Omega} h[\mathbf{z}, \nu] d \mathcal{H}^{N-1}
$$

therefore

$$
\int_{\partial \Omega}(|u-h|-(h-u)[\mathbf{z}, \nu]) d \mathcal{H}^{N-1} \leq 0,
$$

and consequently $[\mathbf{z}, \nu] \in \operatorname{sign}(h-u)$ holds $\mathcal{H}^{N-1}$-a.e. on $\partial \Omega$.

Theorem 2.5. For $v \in B V(\Omega)$, the following conditions are equivalent:

(i) $0 \in \partial \Phi_{h}(v)$.

(ii) $v$ is a solution to problem (2.10). 
Proof. (ii) implies (i): Given $w \in B V(\Omega)$ and applying Green's formula, we obtain

$$
\begin{aligned}
0=\int_{\Omega}(w-v) \operatorname{div} & (\mathbf{z}) d x \\
= & -\int_{\Omega}(\mathbf{z}, D w)+\int_{\Omega}(\mathbf{z}, D v)+\int_{\partial \Omega}[\mathbf{z}, \nu](w-v) d \mathcal{H}^{N-1} .
\end{aligned}
$$

So, having in mind (2.12) and (2.13),

$$
\begin{aligned}
\Phi_{h}(v)=\int_{\Omega}(\mathbf{z}, D v)+ & \int_{\partial \Omega}[\mathbf{z}, \nu](h-v) d \mathcal{H}^{N-1} \\
& =\int_{\Omega}(\mathbf{z}, D w)+\int_{\partial \Omega}[\mathbf{z}, \nu](h-w) d \mathcal{H}^{N-1} \leq \Phi_{h}(w) .
\end{aligned}
$$

Therefore, $0 \in \partial \Phi_{h}(v)$.

(i) implies (ii): We consider problem (2.10). By Theorem 2.4, we obtain $u \in B V(\Omega)$ and $\mathbf{z} \in L^{\infty}\left(\Omega ; \mathbb{R}^{N}\right)$ satisfying (2.11)-(2.13). In particular $-\operatorname{div} \mathbf{z}=0$ in the sense of distributions. Multiplying by $u-v$ and applying Green's formula, it yields

$$
\int_{\Omega}(\mathbf{z}, D(u-v))=\int_{\partial \Omega}[\mathbf{z}, \nu](u-v) d \mathcal{H}^{N-1}
$$

Thus,

$$
\begin{aligned}
\Phi_{h}(v) \leq \Phi_{h}(u)=\int_{\Omega}|D u|+\int_{\partial \Omega} \mid & u-h \mid d \mathcal{H}^{N-1} \\
& =\int_{\Omega}(\mathbf{z}, D v)+\int_{\partial \Omega}[\mathbf{z}, \nu](h-v) d \mathcal{H}^{N-1} .
\end{aligned}
$$

In other words,

$$
\int_{\Omega}(|D v|-(\mathbf{z}, D v))+\int_{\partial \Omega}(|v-h|-[\mathbf{z}, \nu](h-v)) d \mathcal{H}^{N-1} \leq 0 .
$$

Since both integrands are nonnegative, we deduce that $|D v|=(\mathbf{z}, D v)$ as measures and $|v-h|=[\mathbf{z}, \nu](h-v) \mathcal{H}^{N-1}$-a.e. on $\partial \Omega$, so that $[\mathbf{z}, \nu] \in$ $\operatorname{sign}(h-v)$. Since we already have $-\operatorname{div}(\mathbf{z})=0$, we conclude that $v$ is a solution to problem (2.10).

As a corollary of the previous results we finally obtain the first part of Theorem 1.1.

Corollary 2.6. For $v \in B V(\Omega)$ satisfying $\left.v\right|_{\partial \Omega}=h$, the following conditions are equivalent:

(i) $\Phi_{h}(v) \leq \Phi_{h}(u)$ for all $u \in B V(\Omega)$.

(ii) $v$ is a solution to problem (2.10).

(iii) $v$ is a function of least gradient. 
Proof. By Theorem 2.5, we already know that the conditions (i) and (ii) are equivalent.

(i) implies (iii): It is straightforward since $\left.u\right|_{\partial \Omega}=h$ yields

$$
\int_{\Omega}|D v|=\Phi_{h}(v) \leq \Phi_{h}(u)=\int_{\Omega}|D u| .
$$

(iii) implies (i): Fixed $u \in B V(\Omega)$, we have to see that $\Phi_{h}(v) \leq \Phi_{h}(u)$. Given $\epsilon>0$, we apply [4, Lemma 5.5] to get $w \in W^{1,1}(\Omega)$ satisfying

$$
\begin{gathered}
\left.w\right|_{\partial \Omega}=\left.u\right|_{\partial \Omega}-h, \\
\int_{\Omega}|D w| \leq \int_{\partial \Omega}|u-h| d \mathcal{H}^{N-1}+\epsilon, \\
w(x)=0, \quad \text { if } \operatorname{dist}(x, \partial \Omega)>\epsilon .
\end{gathered}
$$

Now consider the function $u-w$. By (2.30), its trace on $\partial \Omega$ is $h$. So we may use (iii) to deduce that

$$
\begin{aligned}
\int_{\Omega}|D v| \leq \int_{\Omega}|D(u-w)| \leq \int_{\Omega}|D u| & +\int_{\Omega}|D w| \\
& \leq \int_{\Omega}|D u|+\int_{\partial \Omega}|u-h| d \mathcal{H}^{N-1}+\epsilon,
\end{aligned}
$$

due to (2.31). Thus,

$$
\Phi_{h}(v)=\int_{\Omega}|D v| \leq \Phi_{h}(u)+\epsilon
$$

and it follows from the arbitrariness of $\epsilon$ that $\Phi_{h}(v) \leq \Phi_{h}(u)$ holds.

2.3. Non-uniqueness. In this subsection we show with an example that uniqueness may be false if we take $h \in L^{1}(\partial \Omega) \backslash C(\partial \Omega)$.

ExAmPle 2.7. Our example is based on [13, Example 3.6], since we will use the function of least gradient appearing there. Let $\Omega=\left\{(x, y) \in \mathbb{R}^{2}\right.$ : $\left.x^{2}+y^{2}<1\right\}$ and consider the boundary datum $h(\theta)=\cos (2 \theta)$. Then the function of least gradient is given by

$$
u(x, y)=\left\{\begin{array}{lll}
2 x^{2}-1, & \text { si }|x|>\frac{\sqrt{2}}{2}, & |y|<\frac{\sqrt{2}}{2} \\
0, & \text { si }|x|<\frac{\sqrt{2}}{2},|y|<\frac{\sqrt{2}}{2} \\
1-2 y^{2}, & \text { si }|x|<\frac{\sqrt{2}}{2},|y|>\frac{\sqrt{2}}{2} .
\end{array}\right.
$$

Applying Corollary 2.6, $u$ is a solution to problem (2.10). Thus, there exists a vector field $\mathbf{z} \in L^{\infty}\left(\Omega ; \mathbb{R}^{N}\right)$, with $\|\mathbf{z}\|_{\infty} \leq 1$, satisfying (2.11) and (2.12).

Let us change a little bit function $h$. The idea is to consider a discontinuous function which has enough roominess so that several functions can be solutions to our problem. 
Consider $h_{1}:[0,2 \pi] \rightarrow \mathbb{R}$ defined by

$$
h_{1}(\theta)= \begin{cases}\cos (2 \theta)+1, & \text { if } \cos (2 \theta)>0 ; \\ \cos (2 \theta)-1, & \text { if } \cos (2 \theta)<0 .\end{cases}
$$

At the points $\frac{\pi}{4}, \frac{3 \pi}{4}, \frac{5 \pi}{4}$ and $\frac{7 \pi}{4}$ which are roots of $\cos (2 \theta)=0$, function $h_{1}$ is not defined, it can take any value.

Then those functions given by

$$
u_{\lambda}(x, y)= \begin{cases}2 x^{2}, & \text { if }|x|>\frac{\sqrt{2}}{2},|y|<\frac{\sqrt{2}}{2} \\ \lambda, & \text { if }|x|<\frac{\sqrt{2}}{2},|y|<\frac{\sqrt{2}}{2} \\ -2 y^{2}, & \text { if }|x|<\frac{\sqrt{2}}{2},|y|>\frac{\sqrt{2}}{2}\end{cases}
$$

with $-1 \leq \lambda \leq 1$, are solutions to problem

$$
\begin{cases}-\operatorname{div}\left(\frac{D u}{|D u|}\right)=0, & \text { in } \Omega ; \\ u=h_{1}, & \text { on } \partial \Omega .\end{cases}
$$

Let us check that the same vector field $\mathbf{z}$ considered above does the job. On account of $(\mathbf{z}, D u)=|D u|$ as measures, we only have to deduce that $\left(\mathbf{z}, D u_{\lambda}\right)=\left|D u_{\lambda}\right|$ as measures. To this end, we take $\varphi \in C_{0}^{\infty}(\Omega)$ and, since $\operatorname{div} \mathbf{z}=0$ it follows that $\left\langle\left(\mathbf{z}, D u_{\lambda}\right), \varphi\right\rangle=-\int_{\Omega} u_{\lambda} \mathbf{z} \cdot \nabla \varphi$, so that we only have to see that

$$
-\int_{\Omega} u_{\lambda} \mathbf{z} \cdot \nabla \varphi=\int_{\Omega} \varphi\left|D u_{\lambda}\right|
$$

Observe that $\Omega$ is divided into three regions: a square and several flaps. We denote

$$
\begin{gathered}
S:=\left\{(x, y) \in \mathbb{R}^{2}: \max \{|x|,|y|\}<\frac{\sqrt{2}}{2}\right\}, \\
F_{x}:=\left\{(x, y) \in \Omega:|x|>\frac{\sqrt{2}}{2}\right\}
\end{gathered}
$$

and

$$
F_{y}:=\left\{(x, y) \in \Omega:|y|>\frac{\sqrt{2}}{2}\right\},
$$

and finally let $\nu_{S}$ be the outward unit vector to $\partial S$. With this notation, the connection between the gradient of $u_{\lambda}$ and that of $u$ is given by

$$
\begin{aligned}
& \int_{\Omega} \varphi\left|D u_{\lambda}\right| \\
& \quad=(1-\lambda) \int_{\partial F_{x}} \varphi d \mathcal{H}^{N-1}+(1+\lambda) \int_{\partial F_{y}} \varphi d \mathcal{H}^{N-1}+\int_{\Omega} \varphi|D u| .
\end{aligned}
$$


On the other hand, we decompose the left hand side of (2.33) and apply Green's formula in $S$ to deduce

$$
\begin{aligned}
& -\int_{\Omega} u_{\lambda} \mathbf{z} \cdot \nabla \varphi=-\int_{S} u_{\lambda} \mathbf{z} \cdot \nabla \varphi-\int_{F_{x}} u_{\lambda} \mathbf{z} \cdot \nabla \varphi-\int_{F_{y}} u_{\lambda} \mathbf{z} \cdot \nabla \varphi \\
& =-\lambda \int_{\partial S} \varphi\left[\mathbf{z}, \nu_{S}\right] d \mathcal{H}^{N-1} \\
& \quad-\int_{F_{x}} \mathbf{z} \cdot \nabla \varphi-\int_{F_{x}} u \mathbf{z} \cdot \nabla \varphi+\int_{F_{y}} \mathbf{z} \cdot \nabla \varphi-\int_{F_{y}} u \mathbf{z} \cdot \nabla \varphi \\
& =-\lambda \int_{\partial F_{x}} \varphi\left[\mathbf{z}, \nu_{S}\right] d \mathcal{H}^{N-1}-\lambda \int_{\partial F_{y}} \varphi\left[\mathbf{z}, \nu_{S}\right] d \mathcal{H}^{N-1} \\
& \quad-\int_{F_{x}} \mathbf{z} \cdot \nabla \varphi+\int_{F_{y}} \mathbf{z} \cdot \nabla \varphi-\int_{\Omega} u \mathbf{z} \cdot \nabla \varphi .
\end{aligned}
$$

Then apply Green's formula in the flaps, taking into account that $\nu_{S}$ is now the unit inward vector, and use the definition of $(\mathbf{z}, D u)$ to get

$$
\begin{aligned}
& -\int_{\Omega} u_{\lambda} \mathbf{z} \cdot \nabla \varphi=(1-\lambda) \int_{\partial F_{x}} \varphi\left[\mathbf{z}, \nu_{S}\right] d \mathcal{H}^{N-1} \\
& \quad+(-1-\lambda) \int_{\partial F_{y}} \varphi\left[\mathbf{z}, \nu_{S}\right] d \mathcal{H}^{N-1}+\langle(\mathbf{z}, D u), \varphi\rangle \\
& =(1-\lambda) \int_{\partial F_{x}} \varphi\left[\mathbf{z}, \nu_{S}\right] d \mathcal{H}^{N-1}-(1+\lambda) \int_{\partial F_{y}} \varphi\left[\mathbf{z}, \nu_{S}\right] d \mathcal{H}^{N-1}+\int_{\Omega} \varphi|D u| .
\end{aligned}
$$

Now, $(\mathbf{z}, D u)\left\llcorner\partial F_{x}=|D u|\left\llcorner\partial F_{x}\right.\right.$ implies

$$
\int_{\partial F_{x}} \varphi\left[\mathbf{z}, \nu_{S}\right] d \mathcal{H}^{N-1}=\int_{\partial F_{x}} \varphi d \mathcal{H}^{N-1}
$$

and similarly it yields

$$
\int_{\partial F_{y}} \varphi\left[\mathbf{z}, \nu_{S}\right] d \mathcal{H}^{N-1}=-\int_{\partial F_{y}} \varphi d \mathcal{H}^{N-1} .
$$

Thus, we obtain that

$$
-\int_{\Omega} u_{\lambda} \mathbf{z} \cdot \nabla \varphi=(1-\lambda) \int_{\partial F_{x}} \varphi d \mathcal{H}^{N-1}+(1+\lambda) \int_{\partial F_{y}} \varphi d \mathcal{H}^{N-1}+\int_{\Omega} \varphi|D u| .
$$

From here, having in mind (2.34), we conclude that (2.33) holds.

REMARK 2.8. Note that all solutions $u_{\lambda}$ have the same frame of superlevel sets. It seems that the boundary datum determines this frame; it obviously not determines a particular solution, unless we deal with continuous functions.

Acknowledgements. The first and third authors have been partially supported by project MTM2012-31103 (Spain) and the second author by projects MTM2010-18128 and MTM2011-27998 (Spain). 


\section{REFERENCES}

[1] L. Ambrosio, N. Fusco and D. Pallara, Functions of Bounded Variation and Free Discontinuity Problems, Oxford Mathematical Monographs, 2000.

[2] F. Andreu, C. Ballester, V. Caselles and J.M. Mazón, The Dirichlet Problem for the Total Variational Flow, J. Funct. Anal. 180, (2001), 347-403.

[3] F. Andreu, V. Caselles, and J.M. Mazón, Parabolic Quasilinear Equations Minimizing Linear Growth Functionals, Progress in Mathematics, vol. 223, 2004. Birkhauser.

[4] G. Anzellotti, Pairings Between Measures and Bounded Functions and Compensated Compactness, Ann. di Matematica Pura ed Appl. IV (135), (1983), 293-318.

[5] E. Bombieri, E. De Giorgi and E. Giusti, Minimal cones and the Bernstein problem. Invent. Math., 7, 243-268 (1969).

[6] M. Giaquinta, G. Modica and J. Soucek, Functionals with linear growth in the calculus of variations I, Comment. Math. Univ. Carolinae 20 (1979), 143-156.

[7] P. Lindqvist Notes on the p-Laplace equation. Report. University of Jyväskylä Department of Mathematics and Statistics, 102.

[8] P. Juutinen, p-harmonic approximation of functions of least gadient. Indiana Univ. Math. J., 54 (2005), 1015-1029.

[9] A. Mercaldo, S. Segura de León and C. Trombetti, On the behaviour of the solutions to p-Laplacian equations as p goes to 1, Publ. Mat. 52, (2008), no. 2, 377-411.

[10] M. Miranda, Sul minimo dell'integrale del gradiente di una funzione, Ann. Scuola Norm. Sup. Pisa, clase Sci., 19 n4 (1965) 627-665.

[11] P. Sternberg, G. Williams and W.P. Ziemer, Existence, uniqueness, and regularity for functions of least gradient. J. Reine Angew. Math. 430, 35-60 (1992).

[12] P. Sternberg and W.P. Ziemer, The Dirichlet problem for functions of least gradient. $\mathrm{Ni}$, Wei-Ming (ed.) et al., Degenerate diffusions. Proceedings of the IMA workshop, held at the University of Minnesota, MN, USA, from May 13 to May 18, 1991. New York: Springer-Verlag. IMA Vol. Math. Appl. 47, 197-214, (1993).

[13] P. Sternberg and W.P. Ziemer, Generalized motion by curvature with a Dirichlet condition. J. Differ. Equations 114, No.2, 580-600, (1994).

J. M. Mazón: Departament D'Anàlisi Matemàtica, Universitat de València, Valencia, Spain.

mazon@uv.es

J. D. Rossi: Depto. de Análisis Matemático, Universidad de Alicante, Ap. Correos 99, 03080, Alicante, Spain, and Depto. de Matemática, FCEyN UBA Ciudad Universitaria, Pab 1 (1428) Buenos Aires, Argentina.

julio.rossi@ua.es

S. Segura de León: Departament d'Anàlisi Matemàtica, Universitat de València, Valencia, Spain.

sergio.segura@uv.es 